INPLASY

PROTOCOL

To cite: LI et al. Xueshuantong injection in treating Deep Venous Thrombosis: a systematic review and experimental sequential analysis. Inplasy protocol 2020120117. doi: 10.37766/inplasy2020.12.0117

Received: 24 December 2020

Published: 24 December 2020

Corresponding author: Weijing Fan

18811023202@163.com

Author Affiliation:

Shuguang Hospital Affiliated to Shanghai University of Traditional Chinese Medicine

Support: No.81774310; 81804096.

Review Stage at time of this submission: The review has not yet started.

Conflicts of interest:

None.

\section{Xueshuantong injection in treating Deep Venous Thrombosis: a systematic review and experimental sequential analysis}

LI, W1; Xu, F²; Fan, W3; Liu, G4; Xu, L5; Wang, X6; Lu, H77 LI, Y8.

Review question / Objective: This systematic review aimed to assess the efficacy and safety of Xueshuantong injection in the treatment of deep vein thrombosis (DVT) systematically, and provide evidence-based reference for clinical treatment. Condition being studied: Current evidence regarding the efficacy and safety of Xueshuantong injection for Deep Venous Thrombosis (DVT) is controversial.

Information sources: Chinese database: Chinese National Knowledge Infrastructure Database; the Chongqing VIP Database; Chinese Biomedical Database; Wanfang Database. English databases: PubMed, Cochrane Library, Web of knowledge, Embase, MEDLINE.

INPLASY registration number: This protocol was registered with the International Platform of Registered Systematic Review and Meta-Analysis Protocols (INPLASY) on 24 December 2020 and was last updated on 24 December 2020 (registration number INPLASY2020120117).

\section{INTRODUCTION}

Review question / Objective: This systematic review aimed to assess the efficacy and safety of Xueshuantong injection in the treatment of deep vein thrombosis (DVT) systematically, and provide evidence-based reference for clinical treatment.

Condition being studied: Current evidence regarding the efficacy and safety of Xueshuantong injection for Deep Venous Thrombosis (DVT) is controversial. 


\section{METHODS}

Participant or population: patients were in line with the relevant diagnosis of deep vein thrombosis.

Intervention: Xueshuantong injection or Xueshuantong combined with conventional treatment.

Comparator: Conventional treatment or placebo.

Study designs to be included: RCTs.

Eligibility criteria: Xueshuantong injection in treating Deep Venous Thrombosis; RCT.

Information sources: Chinese database: Chinese National Knowledge Infrastructure Database; the Chongqing VIP Database; Chinese Biomedical Database; Wanfang Database. English databases: PubMed, Cochrane Library, Web of knowledge, Embase, MEDLINE.

Main outcome(s): Clinical effective rate.

Additional outcome(s): Incidence rate of adverse events.

Quality assessment / Risk of bias analysis: Quality evaluation tool: bias risk Assessment tool developed by Cochrane collaboration Network.

Strategy of data synthesis: We plan to perform data analysis using RevMan V.5.4 provided by the Cochrane Collaboration. We plan to present the dichotomous outcomes with risk ratios and $95 \%$ Cls. We will report estimates for continuous data as weighted mean difference and $95 \%$ Cls. Standardised mean difference will be used if different measurement tools were used in different trials.

Subgroup analysis: If we find substantial heterogeneity (when the I'statistic exceeds $50 \%$ ), and there are sufficient data, we will investigate the possible causes by exploring the impact of the condition of the individuals and interventions (i.e. participant characteristics, addition of adjuvant therapies) using subgroup analyses.

Sensibility analysis: We will perform a sensitivity analysis to determine whether our results are robust by excluding those studies assessed as having a high risk of bias. The following sensitivity analyses will be performed to test whether critical methodological factors or decisions have affected the main result, where there is a sufficient number of studies in the metaanalyses: removing unpublished data changing effects model.

Country(ies) involved: China.

Keywords: Xueshuantong; deep venous thrombosis; experimental sequential analysis.

Contributions of each author:

Author 1 - Weihui LI.

Author 2 - Feng Xv.

Author 3 - Weijing Fan.

Author 4 - Guobin Liu.

Author 5 - Lei Xu.

Author 6 - Xvhong Wang.

Author 7 - Huimin Lu.

Author 8 - Yuanxiang LI. 